\title{
Genomic analysis of childhood hearing loss in the Yoruba population of Nigeria
}

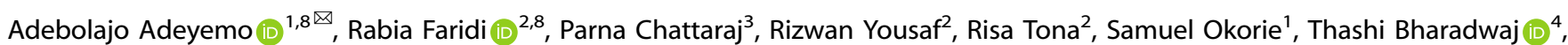 \\ Liz M. Nouel-Saied ${ }^{4}$, Anushree Acharya ${ }^{4}$, Isabelle Schrauwen (iD ${ }^{4}$, Robert J. Morell ${ }^{5}$, Suzanne M. Leal (D) ${ }^{4,6}$, Thomas B. Friedman (D) ${ }^{2}$, \\ Andrew J. Griffith (iD ${ }^{3,7}$ and Isabelle Roux (iD ${ }^{3 \times}$
}

This is a U.S. government work and not under copyright protection in the U.S.; foreign copyright protection may apply 2021

\begin{abstract}
Although variant alleles of hundreds of genes are associated with sensorineural deafness in children, the genes and alleles involved remain largely unknown in the Sub-Saharan regions of Africa. We ascertained 56 small families mainly of Yoruba ethno-lingual ancestry in or near Ibadan, Nigeria, that had at least one individual with nonsyndromic, severe-to-profound, prelingual-onset, bilateral hearing loss not attributed to nongenetic factors. We performed a combination of exome and Sanger sequencing analyses to evaluate both nuclear and mitochondrial genomes. No biallelic pathogenic variants were identified in GJB2, a common cause of deafness in many populations. Potential causative variants were identified in genes associated with nonsyndromic hearing loss (CIB2, COL11A1, ILDR1, MYO15A, TMPRSS3, and WFS1), nonsyndromic hearing loss or Usher syndrome (CDH23, MYO7A, PCDH15, and USH2A), and other syndromic forms of hearing loss (CHD7, OPA1, and SPTLC1). Several rare mitochondrial variants, including m.1555A $>\mathrm{G}$, were detected in the gene MT-RNR1 but not in control Yoruba samples. Overall, 20 (33\%) of 60 independent cases of hearing loss in this cohort of families were associated with likely causal variants in genes reported to underlie deafness in other populations. None of these likely causal variants were present in more than one family, most were detected as compound heterozygotes, and $77 \%$ had not been previously associated with hearing loss. These results indicate an unusually high level of genetic heterogeneity of hearing loss in Ibadan, Nigeria and point to challenges for molecular genetic screening, counseling, and early intervention in this population.
\end{abstract}

European Journal of Human Genetics (2022) 30:42-52; https://doi.org/10.1038/s41431-021-00984-w

\section{INTRODUCTION}

Hearing loss $(\mathrm{HL})$ is one of the most common sensory disorder worldwide. In high-income countries (HICs), the estimated prevalence of permanent bilateral $\mathrm{HL}$ of $40 \mathrm{~dB} \mathrm{HL}$ or more is estimated to be between 1.33 and 1.5 per 1000 live births; 2.7 per 1000 by 5 years of age [for review [1, 2]]. HL seems to be more prevalent in Sub-Saharan Africa [3]. In Nigeria, the exact prevalence of $\mathrm{HL}$ (as defined by $>40 \mathrm{~dB} \mathrm{HL}$ ) is unknown but has been estimated to be at least 19.2 per 1000 newborns [4-6].

Early childhood HL in HICs is thought to be primarily due to genetic factors and monogenic variants, although permanent profound $\mathrm{HL}$ can also be caused by environmental factors, or a combination of both [7, 8]. In Western Africa and Nigeria, environmental factors have been reported as frequent risk factors for HL [3, 9-12]. There are only a few published studies of genetic contributions to HL in Sub-Saharan African populations [13-15]. In many populations, pathogenic variants of GJB2 [MIM: 121011] sometimes in trans with deletions in the GJB2/GJB6 genomic region (del(GJB6-D13S1830), del(GJB6-D13S1854), and del(chr13:19,837,
$344-19,968,698)[16,17]$ represent a common cause of genetic HL. Variants in these genes have rarely been found in individuals with HL from Sub-Saharan Africa or of Sub-Saharan African descent, except in Ghana where the pathogenic variant NM_004004.6: c.427C >T p.(Arg143Trp) of GJB2 is prevalent [13]. Pathogenic variants in other genes associated with HL such as SLC26A4 [MIM: 605646], OTOF [MIM: 603681], HGF [MIM: 142409], MYO15A [MIM: 602666], and TMC1 [MIM: 606706] are found frequently in specific populations outside of Sub-Saharan Africa. It is still unclear whether pathogenic variants in these same genes or others are associated with $\mathrm{HL}$ in populations of Sub-Saharan Africa, and whether some of these pathogenic variants are common.

Nigeria is located in Sub-Saharan West Africa and is the most populous country in Africa with approximately 210 million inhabitants. Our cohort is comprised of families from Ibadan, Nigeria, with at least one member with nonsyndromic congenital or early childhood severe-to-profound $\mathrm{HL}$ without a known environmental cause. We used exome and Sanger sequencing analyses in these subjects to study nuclear and mitochondrial

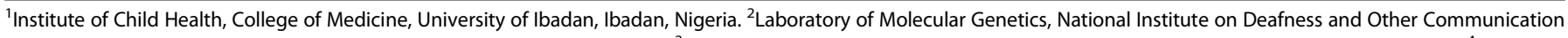

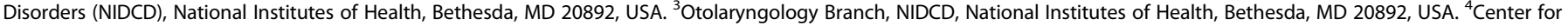

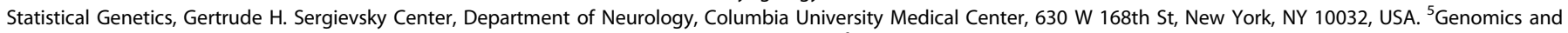

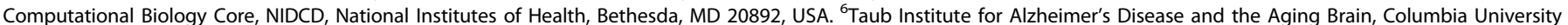

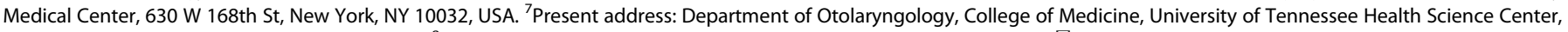

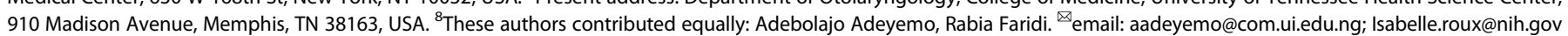


genes reported to be associated with human nonsyndromic or syndromic $\mathrm{HL}$ in other populations, and identify potential causative variants associated with $\mathrm{HL}$ in Yoruba ethnic group.

\section{MATERIALS AND METHODS Study approval}

This study was approved by the University of Ibadan, University College Hospital Ethics Committee (UI/EC/15/0047), the Combined Neurosciences Institutional Review Board at the National Institutes of Health (01-DC-0229), and the Institutional Review Board of Columbia University (IRB-AAAS2343). All adult participants provided written informed consent. For minors, at least one parent provided consent and minors provided their assents.

\section{Subjects, recruitment, and ascertainment}

Fifty-six families were studied, which included 70 individuals with $\mathrm{HL}$ and 102 individuals without HL (Supplementary Table 1). Probands were identified from government schools for the deaf or from the Deaf Community in Ibadan, southwest Nigeria. The majority of subjects were of the Yoruba ethnic origin, the most prevalent ethnic group in southwest Nigeria. All probands were of Black African ancestry with bilateral congenital or prelingual-onset nonsyndromic HL. Whenever possible, parents, siblings, and other relatives of probands were enrolled. Individuals with $\mathrm{HL}$ associated with obvious features of known syndromes or whose $\mathrm{HL}$ was likely secondary to a nongenetic etiology such as trauma, infection, metabolic, or immunologic disorders, or exposure to ototoxic agents such as noise or aminoglycoside antibiotics were excluded from the study. None of the probands had a reported history of dizziness or significant neonatal illness. Physical examinations were carried out by a physician (AA) to identify or rule out obvious syndromic features. Pure-tone audiometry was performed, in some cases, to confirm bilateral severe-to-profound sensorineural HL. Family history was obtained by interview of all participants.

\section{DNA sequencing analyses}

Genomic DNA (gDNA) was extracted from peripheral blood samples and processed as shown in Fig. 1. We first screened whole gDNA extract from one subject with $\mathrm{HL}$ from each family, or both parents if they both had $\mathrm{HL}$ $(N=60$ independent cases tested), by Sanger sequencing the single protein-coding exon of GJB2, NM_000601.6:C.482+1986_1988delTGA and c.482+1991_2000delGATGATGAAA intronic deletions of HGF that are frequent in Asia [18] and the mitochondrial genes MT-RNR1, MT-TS1, and MT-TL1 using custom designed primers (Supplementary Table 2). Exome sequencing (ES) was performed with gDNA samples from 67 individuals with $\mathrm{HL}$ and 48 family members without reported HL. ES libraries were prepared using a Nextera Rapid Capture Exome kit (Illumina, San Diego, CA, USA) and sequenced on an Illumina NextSeq500 instrument. The mean depth of coverage of the targeted coding regions was $44 \times$. Due to insufficient coverage, microRNA MIR96 [MIM: 611606, (DFNA50)] was Sanger-sequenced [19].

\section{Data analyses}

Sequence data from exome libraries were mapped to the GRCh38 human reference genome using the bcbio-nextgen germline variant calling pipeline. See Supplementary Information 1 for all URLs and corresponding references. Reads were mapped using the Burrows-Wheeler Aligner (BWA$M E M)$, then remapped after removing duplicate data and recalibration with Genome Analysis Toolkit (GATK). Single-nucleotide variants (SNV) and insertion/deletions were detected using GATK-haplotypecaller, platypus, varscan, freebayes, and samtools variant callers. The final ensemble variant call file (vcf) required concordance between at least two callers. As a quality control, we checked the entire cohort for excess heterozygosity and verified the sex of each individual with exome data using PLINKv1.9. Family relationships were verified via both Identity-by-Descent sharing (PLINKv1.9) and Kinship-based INference for Gwas (KING) algorithm. Samples identified to have problems were removed from further exome data analysis. Individual vcf files were then analyzed using the Ingenuity Variant Analysis platform (IVA version 5.6, Qiagen, Hilden, Germany) using data from gnomAD v2.1.1, The Human Gene Mutation Database (HGMD) v2019.2, NCBI dbSNP v151, and Allele Frequency Community (AFC) v201909-25. The systematic review of the variants and prioritization of the predicted pathogenic, likely pathogenic, and variants of uncertain significance (VUS) with predicted deleterious effects segregating in each family was performed as shown in Fig. 1 and is summarized below. Copy number variants (CNVs) were assessed using the copy number inference from exome reads (CoNIFER) pipeline.

\section{Variant prioritization and verification}

Single-nucleotide and insertion-deletion variants detected in at least eight reads from any one individual with $\mathrm{HL}$, and absent from the top $5 \%$ of most variable 100-bp exonic sequences in 1000 Genomes database, were further analyzed. We first searched for variants in genes associated with nonsyndromic HL likely to be pathogenic (Supplementary Information 2) assuming complete penetrance. In Family 29, HL was present in both parents and children. In most families, only one individual with $\mathrm{HL}$ was present and the family history was limited. Variants were analyzed and evaluated according to several inheritance models (Fig. 1). In order to avoid missing prevalent and potentially enriched pathogenic alleles (founder variants), we used thresholds that were larger, by a factor of ten, than conventional thresholds for minor allele frequency for $\mathrm{HL}$ [20]. Assuming a model of autosomal dominant (AD) inheritance and searching for de novo variants, variants were only considered when: (1) they were already established to be pathogenic or likely pathogenic, (2) they were not detected in unaffected parents, or (3) when the ES data from one or both parents were not available, their minor allele frequency (MAF) was $\leq 0.5 \%$ in any reference subpopulation in gnomAD, ExAC, and in African (American) from the National Heart, Lung, and Blood Institute Exome Sequencing Project Exome Variant Server. In models of autosomal recessive (AR) and X-linked inheritance (XL), only variants with a frequency of less than $5 \%$ in the variant databases were initially considered, unless they were an established pathogenic or likely pathogenic variant. Variants detected in family members without reported $\mathrm{HL}$ that were either homozygous or heterozygous in cis without additional variant in trans were disregarded. Previously reported pathogenic variants, novel nonsense or frameshift variants, splice site variants within two nucleotides of intron-exon junctions, and missense variants with a combined annotation-dependent depletion (CADD) score of at least 15 were reported and further analyzed, as well as synonymous variants predicted to affect splicing (Supplementary Table 3). Variants were prioritized for further study when: (1) one of them had an allele frequency $\leq 1 \%$ (recessive model); (2) there were five or less (recessive model) or no (dominant model) individuals homozygous for the variant reported in gnomAD; and (3) if at least two algorithms predicted the variant to be damaging/deleterious or likely damaging/deleterious. The algorithms used were SIFT, PolyPhen-2, FATHMMMKL or XF, MutationAssessor, MutationTaster, REVEL, and CADD (Supplementary Information 1). Evolutionary conservation of nucleotides was evaluated by PhyloP. The effects of variants on mRNA splicing were predicted by MaxEntScan, Human splice finder, BDGP Splice Site Prediction, and NetGene2.

In addition, a second and independent SNV/insertion/deletion annotation and prioritization analysis was performed with the same outcome, on the jointly called variants with GATK only, similar to what has been described in detail [21]. In short, variants were annotated using ANNOVAR, including prediction scores from dbnsfp35a and dbscSNV1.1, ClinVar, and several frequency databases such as gnomAD. Variants were considered further if either (1) they were reported pathogenic/likely pathogenic in ClinVar; or (2) they met filtering criteria including inheritance model (AR, $A D$, and $X L$ [including de novo]), variant location (exonic and splice site), predicted effect (missense, nonsense, frameshift, and in-frame insertion/ deletion, splicing and start and stop altering), and variant frequency ( $<0.5 \%$ MAF for AR and $\mathrm{XL} ;<0.05 \% \mathrm{MAF}$ for $\mathrm{AD})$ [21].

Prioritized variants were validated via Sanger sequencing and further tested for co-segregation with $\mathrm{HL}$ in each family. Variant frequency in the Yoruba population was determined using Ensembl to access data from 108 Yoruba individuals from Ibadan, Nigeria (AFR YRI) available through 1000 Genomes. When a variant site was not evaluated due to insufficient read depth for example, we performed Sanger sequencing using gDNA samples from 118 unrelated Yoruba individuals (59 females and 59 males) from Ibadan, Nigeria (NHGRI Repository at the Coriell Institute for Medical Research Cat \# MGP00013). Variants were then classified according to the American College of Medical Genetics and Genomics/Association for Molecular Pathology (ACMG/AMP) Guidelines for the interpretation of sequence variants in $\mathrm{HL}$ genes [22], taking into account data from ClinVar, the Deafness Variation Database, and HGMD, with two modifications. The genetic causes of $\mathrm{HL}$ have not yet been well characterized in the YRI population, and the information regarding variant MAF in this population is still limited, so we did not exclude any variant based on their "high" MAF. PP3 criterion was applied even if the REVEL score was below 0.7, if at least two of the algorithms used predicted that the variant was damaging or likely damaging (Fig. 1 and Table 1). In families with potential de novo variants, maternity and paternity were verified by genotyping short tandem repeat markers when no 
None found

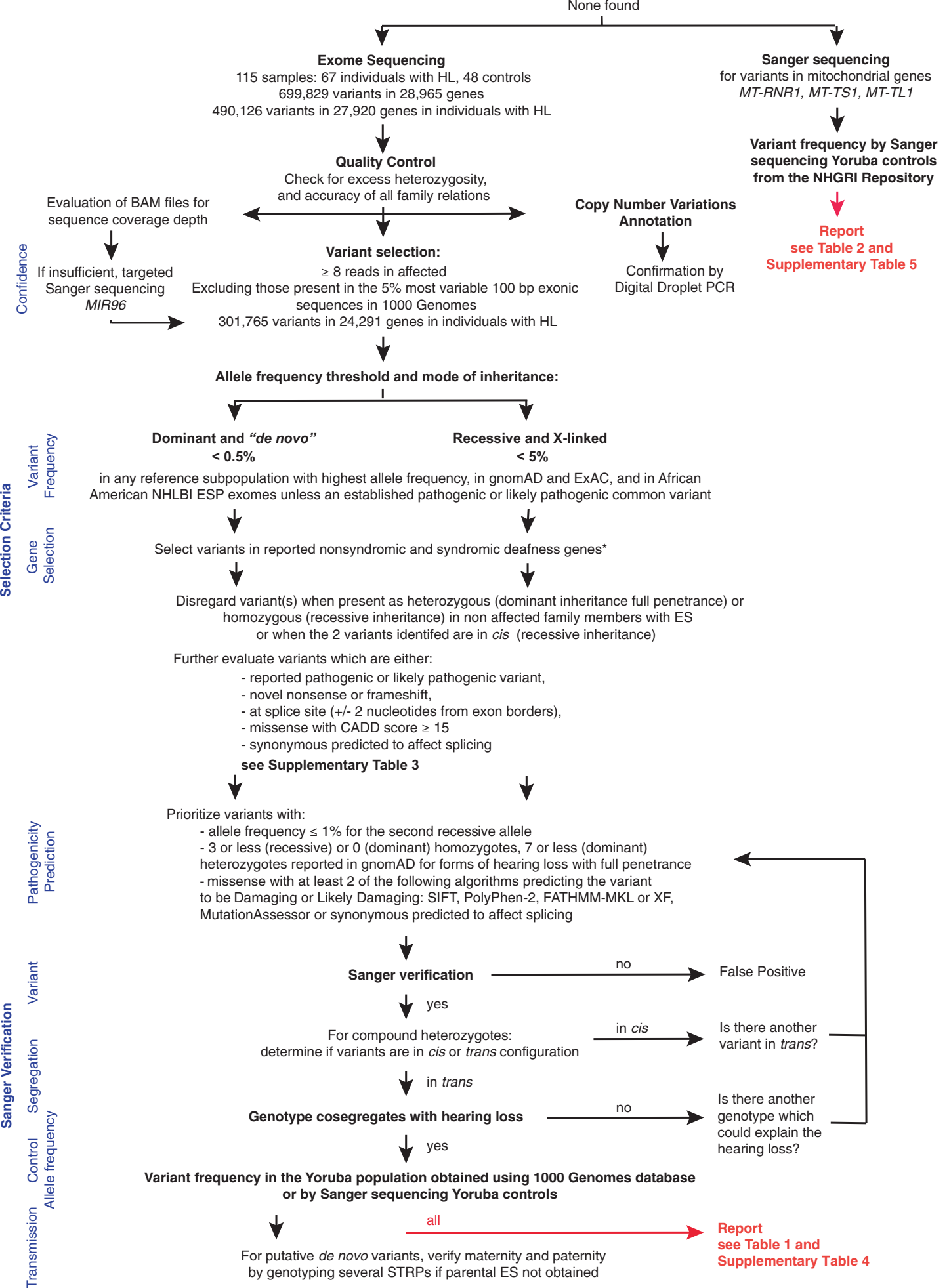

Fig. 1 Experimental and analysis pipeline used to identify potential causative variants in reported genes associated with nonsyndromic and syndromic forms of hearing loss. Diagram shows the experimental strategy used for processing and analyzing the samples and data. Sanger sequencing was done on the gDNA of both parents if they had HL or on the gDNA of the proband with HL otherwise, for a total of 60 independent cases of HL. Variant counts are shown for the initial steps. The lists of genes included for these analyses are presented in Supplementary Information 2 and 3. *Full penetrance of $\mathrm{HL}$ was assumed unless reported otherwise for some genes associated with syndromic forms of $\mathrm{HL}$. STRP short tandem repeat polymorphisms. 


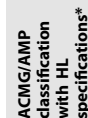

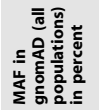

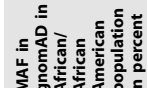

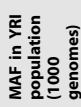

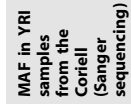

ปิे

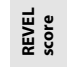

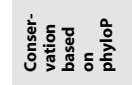

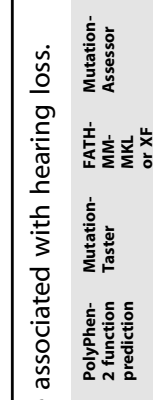



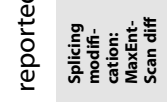

嵌

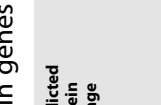

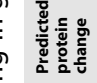

离

斊

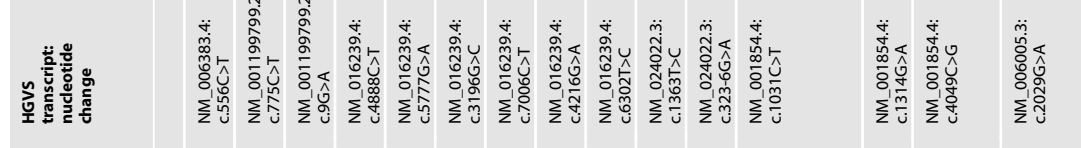

吾

竞

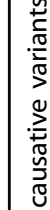

ते

宊

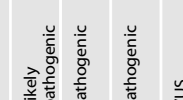





官

$$
\stackrel{\frac{9}{\pi}}{\frac{1}{2}} \frac{2}{2}
$$

$\frac{2}{\sqrt[2]{2}}$

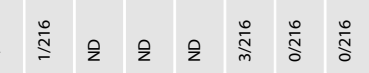

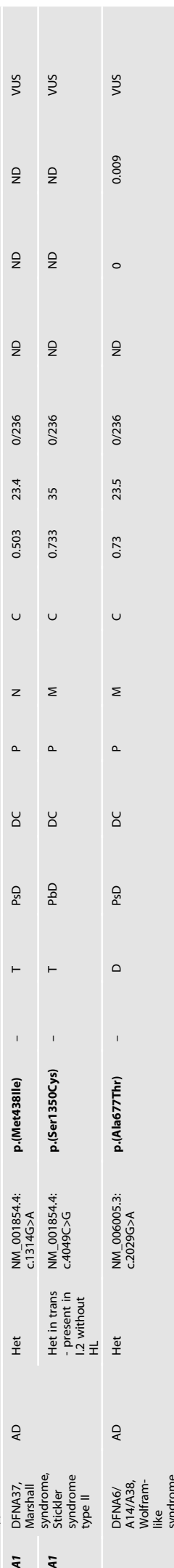

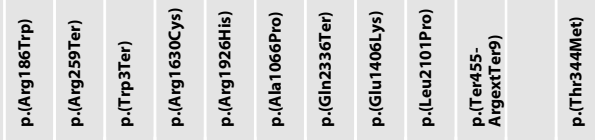

$\frac{\circ}{2} \frac{\circ}{\frac{\circ}{2}} \infty \frac{\circ}{\frac{\circ}{2}}$

$\frac{8}{2}$

$\frac{2}{2}$

$\circ$

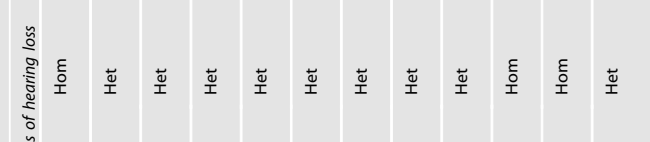

高

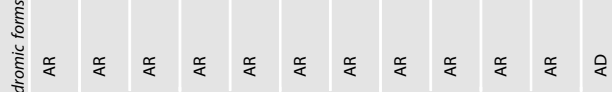

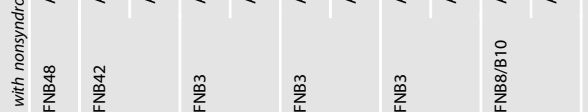

密

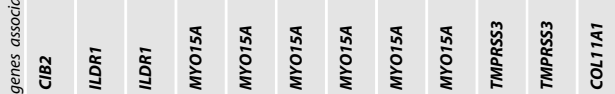



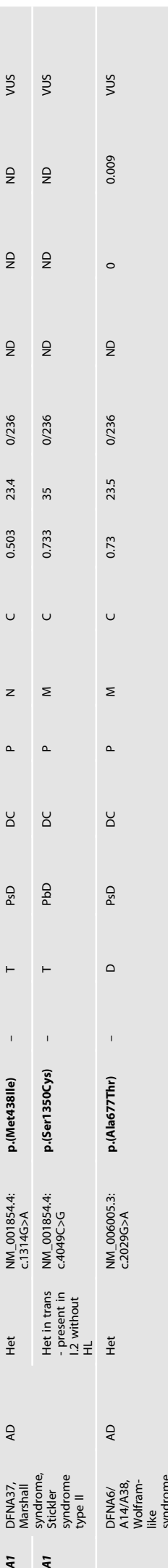

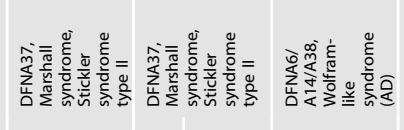

₹

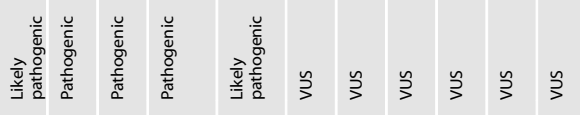

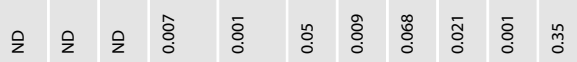

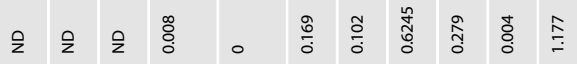

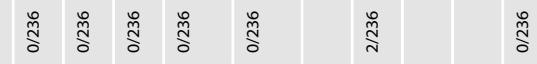

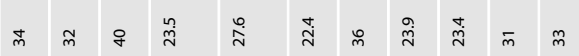

, , 商 兽

ப

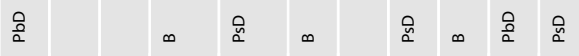

, $\frac{\infty}{\infty}, 1, \mathbb{\infty}_{0}^{\infty} \frac{\infty}{0}$

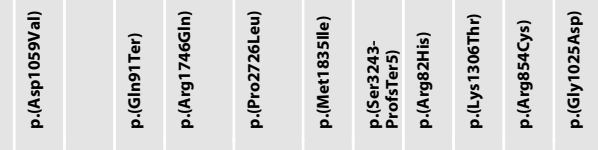

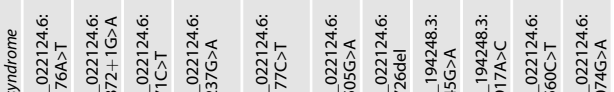

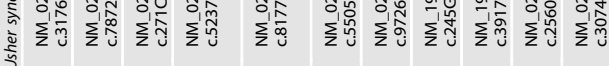


हैํํำ

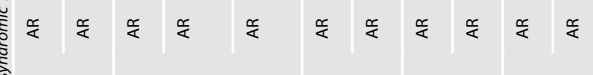

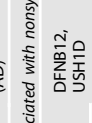



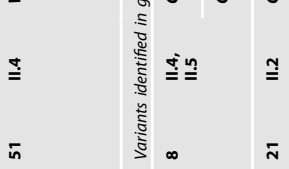




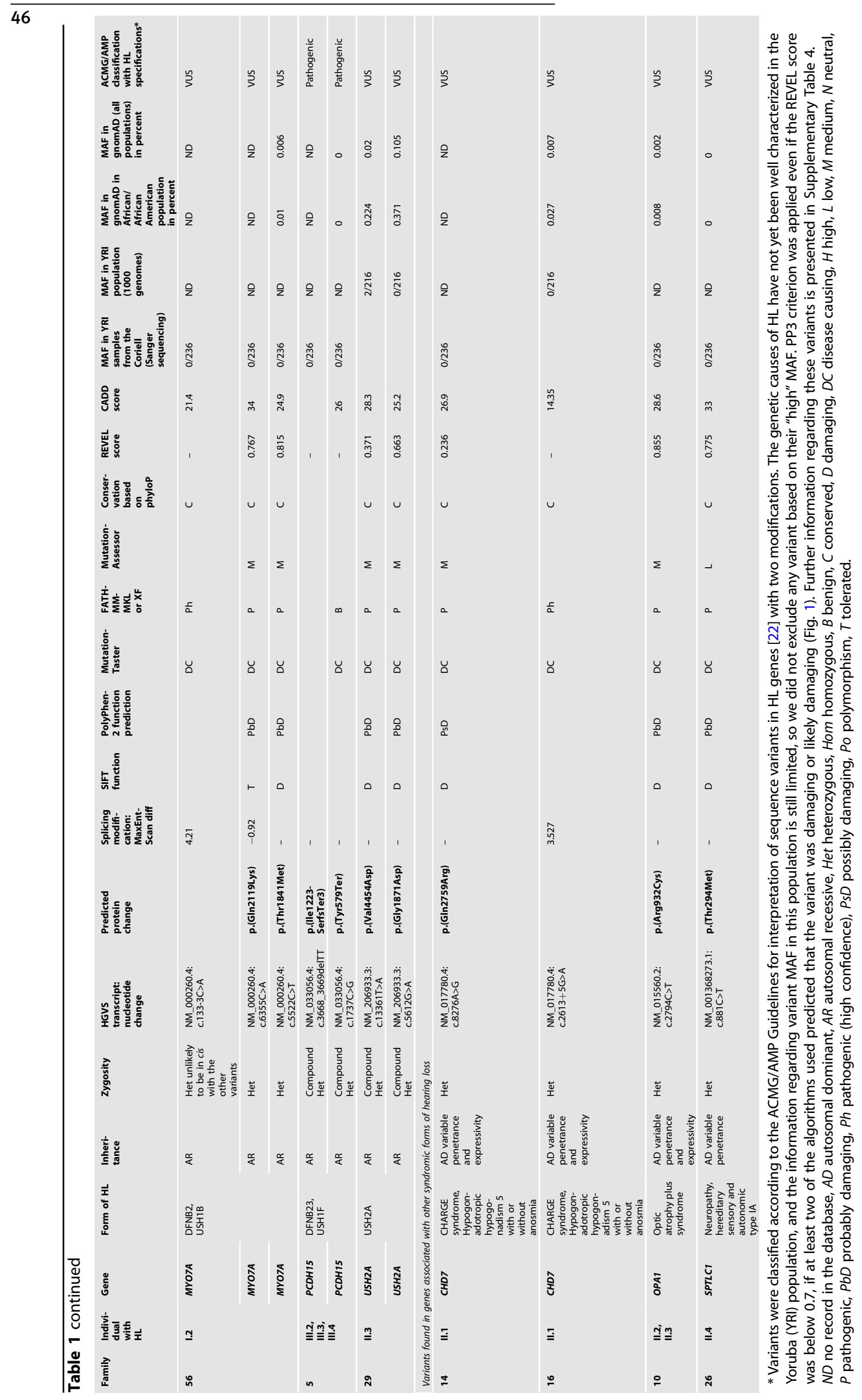


a

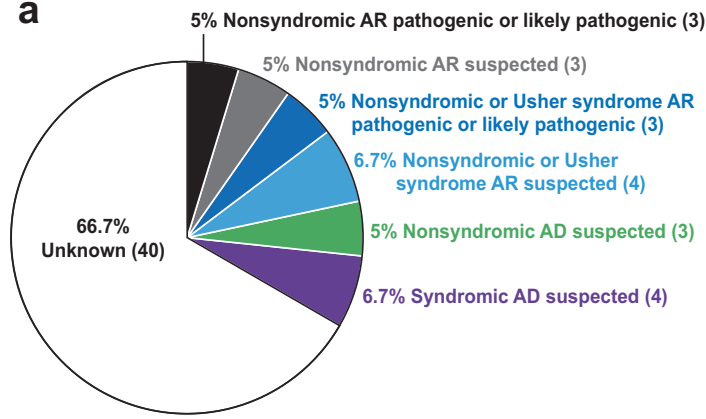

b

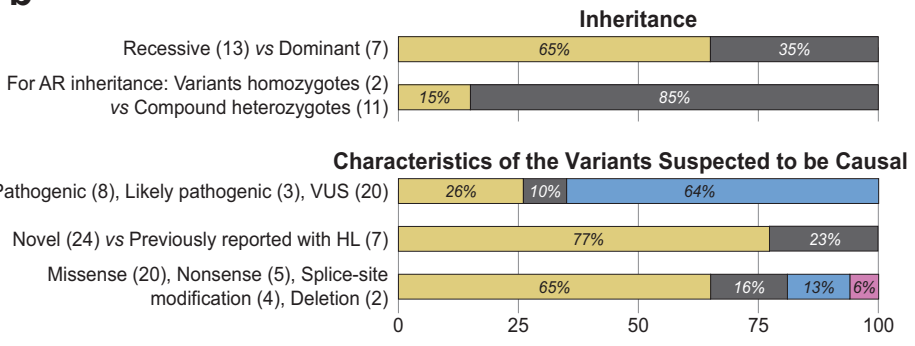

C

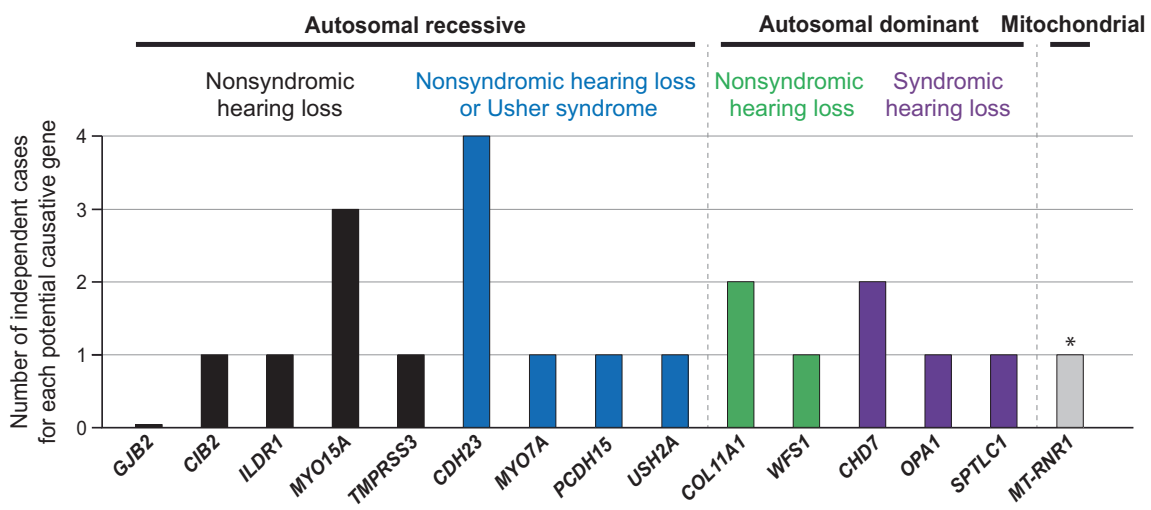

Fig. 2 Etiology of hearing loss in a cohort of 56 Nigerian Yoruba families with $\mathbf{6 0}$ independent cases of hearing loss. a Relative distribution of the likely genetic causes of HL identified in Yoruba individuals according to ACMG/AMP criteria for interpretation of sequence variants in HL genes, mode of inheritance, and gene classification. AR autosomal recessive, AD autosomal dominant. The number of cases per category is indicated in parentheses. b Characteristics of the cases and variants identified. c Distribution of the cases as a function of the genes in which potential causative variants were identified. The associated modes of inheritance are indicated. ${ }^{*} M T-R N R 1$ variant $\mathrm{m} .1555 \mathrm{~A}>\mathrm{G}$ was found in an individual with HL also carrying potential causative VUS in CDH23 and OTOF (the latter were not indicated here but are available in Table 1).

parental ES data were available. A similar analysis was performed for genes implicated in syndromic forms of $\mathrm{HL}$ assuming full penetrance unless otherwise reported [23].

CNVs were annotated using ANNOVAR, BioMart database, and bedtools to obtain genes, functions, and known disease associations [21]. Rare variants $(\mathrm{MAF}<0.5 \%)$ were selected based on variant frequency data from the Database of Genomic Variants and gnomAD. Candidate CNVs were visually inspected with the CoNIFER plotting tool and were subsequently validated using Digital Droplet PCR (ddPCR, BioRad Laboratories, Hercules, CA, USA) [24] if deemed of interest. ddPCR was also used to confirm the absence of a deletion in trans when a damaging variant appeared to be homozygous and gDNA of one parent was not available.

\section{RESULTS}

\section{Study subjects and families}

In the 56 families studied from Ibadan, Nigeria, a total of $60(N)$ independent cases of $\mathrm{HL}$ were studied (Supplementary Table 1). All individuals with $\mathrm{HL}$ had bilateral congenital or prelingual-onset severe-to-profound $\mathrm{HL}$ and used sign language. The presence of pathogenic, likely pathogenic and VUS with predicted deleterious effects in genes reported to be associated with $\mathrm{HL}$ was investigated (Fig. 1).

\section{GJB2 pathogenic variants are not prevalent in the Yoruba population of Nigeria}

Sanger sequence analyses of GJB2 in 60 independent cases with $\mathrm{HL}$ revealed in one subject a heterozygous pathogenic recessive variant that was previously reported (NM_004004.6:c.405delC, p.(Tyr136fsTer32)). One heterozygous previously reported VUS was also identified in the $5^{\prime}$ UTR of GJB2 (c. $-6 T>A$ ) in three individuals from three different families. We excluded by PCR testing the possibility that a second allele involving deletions at the $D F N B 1 A / B$ locus [del (GJB6-D13S1830), del(GJB6-D13S1854), and del(chr13:19,837,344$19,968,698)]$ was in trans in any of these four subjects who carry heterozygous GJB2 variants.

\section{Exome sequencing reveals high heterogeneity in potential causal variants in genes associated with hearing loss} ES was performed using gDNA samples from 67 individuals with $\mathrm{HL}$ and a subset of family members without $\mathrm{HL}$ (48). The region encoding microRNA MIR96 was Sanger-sequenced, but no variants were identified. The small size of the families and the absence of reported consanguinity precluded linkage or homozygosity mapping studies. Lack of access to one or both parents in several families limited our ability to confirm that identified heterozygous variants were compound heterozygous.

Potential causative variants were found in 20 of 60 independent cases of $\mathrm{HL}$ of our cohort (Table 1 and Supplementary Table 4). These variants were identified in genes associated with nonsyndromic forms of $\mathrm{HL}$ in nine cases, in genes associated with nonsyndromic forms of $\mathrm{HL}$ or Usher syndrome in seven cases, and in genes associated with other forms of syndromic HL in four cases (Fig. 2a, c). Pathogenic or likely pathogenic variants were only identified in 6 of 20 independent cases of $\mathrm{HL}$ in our cohort (Table 1, Supplementary Table 4, and Fig. 2a). Sixteen percent of these variants were nonsense variants, $6 \%$ were small deletions, $13 \%$ were predicted to affect splicing, and $65 \%$ were missense variants (Fig. 2b). Seventy-seven percent of these variants had not been previously associated with $\mathrm{HL}$. We also identified several potential CNVs. However, subsequent ddPCR experiments either did not confirm the presence of these CNVs or showed that they did not cosegregate with the HL. 
a

CIB2: c.556C>T p.(Arg186Trp)

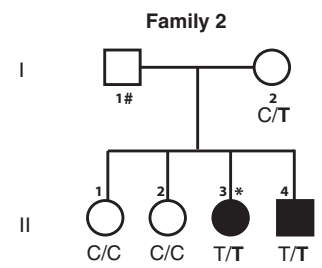

MYO15A: c.4216G>A p.(Glu1406Lys) MYO15A: c.6302T>C p.(Leu2101Pro)

Family 44

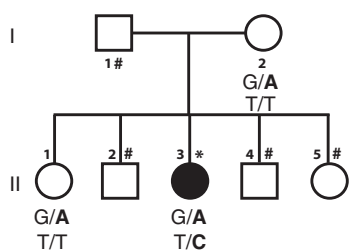

COL11A1: c.1031C>T p.(Thr344Met)

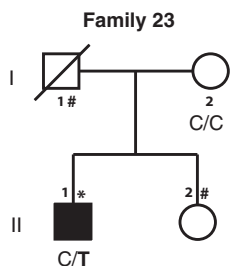

b

CDH23: c.3176A >T p.(Asp1059Val) CDH23: c. $7872+1 \mathrm{G}>\mathrm{A}$

Family 8

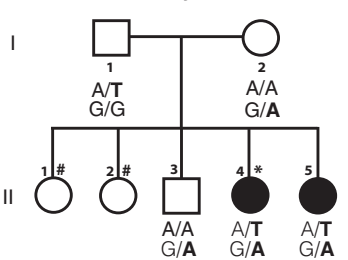

MYO15A: c.4888C>T p.(Arg1630Cys) MY015A: c.5777G>A p.(Arg1926His) Family 6

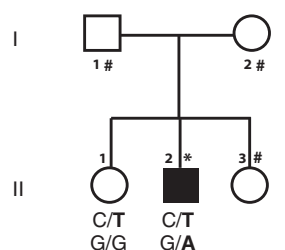

TMPRSS3: c.323-6G>A

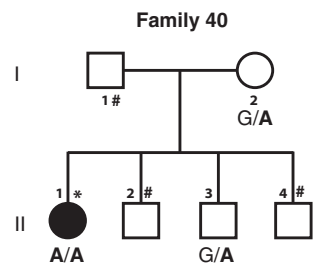

COL11A1: c.1314G>A p.(Met438lle)

Family 32

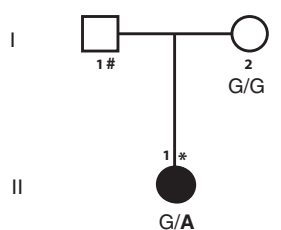

CDH23: c.271C>T p.(GIn91Ter) CDH23: c.5237G>A p.(Arg1746GIn)

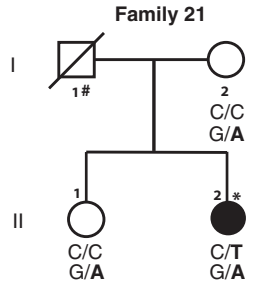

MYO15A: c.3196G>C p.(Arg1066Pro) MYO15A: c.7006C>T p.(GIn2336Ter)

Family 13

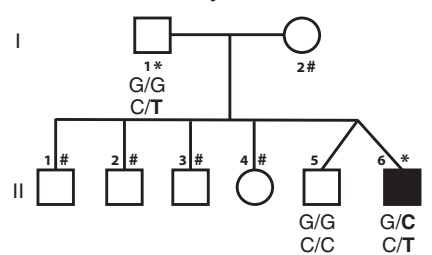

male female

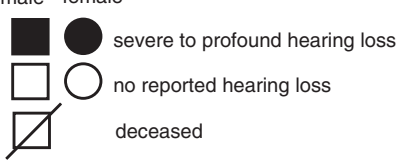

* exome sequenced

\# gDNA sample unavailable

+ wild-type allele

WFS1: c.2029G>A p.(Ala677Thr)

Family 51

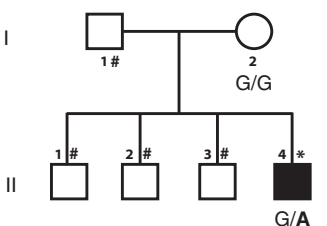

CDH23: c.5505G>A p.(Met1835lle) CDH23: c.9726delC p.(Ser3243ProfsTer5)

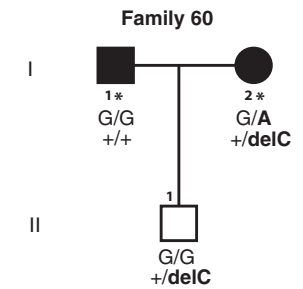

PCDH15: c.1737C>G p.(Tyr579Ter) PCDH15: c.3668_3669delTT p.(Ile1223SerfsTer3) USH2A: c.5612G>A p.(Gly1871Asp) USH2A: c.13361T>A p.(Val4454Asp)
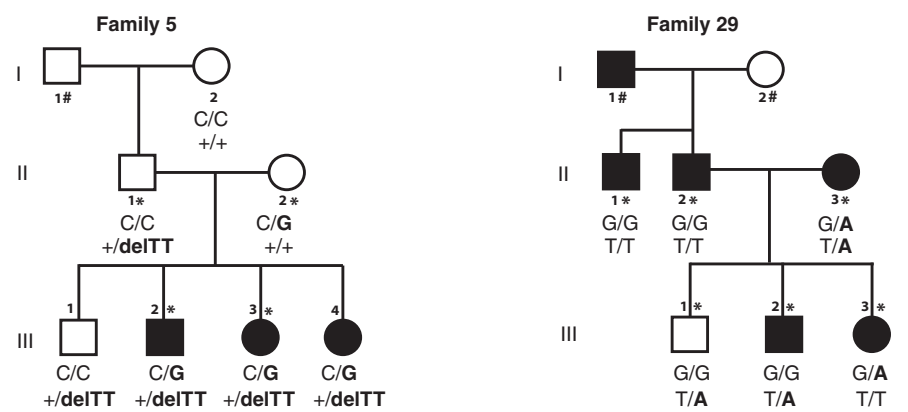

Fig. 3 Pedigrees of Nigerian Yoruba families segregating likely causative variants in genes associated with nonsyndromic hearing loss and Usher syndrome. a Families with variants in genes associated with nonsyndromic hearing loss. b Families with variants in genes associated with nonsyndromic hearing loss or Usher syndrome. Circles represent females and squares indicate males. Solid symbols represent individuals with bilateral severe-to-profound $\mathrm{HL}$; non-shaded symbols represent unaffected individuals; crossed symbols represent deceased individuals. Individuals studied by exome sequencing are indicated by an asterisk, \# indicates individuals whose gDNA was not available. The genotype for the candidate variant(s) are shown above each pedigree. + represents the reference allele; /, variants in trans. Only pedigrees with segregation information are presented. 
CHD7: c.8276A>G p.(Gln2759Arg)

Family 14

I

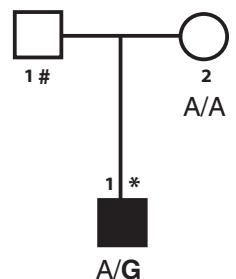

CHD7: c. $2613+5 G>A$

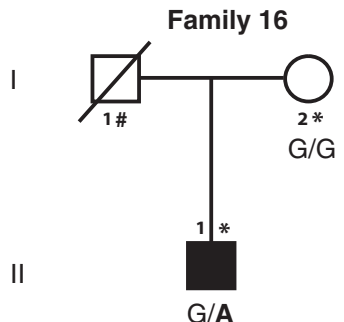

OPA1: c.2794C>T p.(Arg932Cys)



SPTLC1: c.881C>T p.(Thr294Met)

Family 26

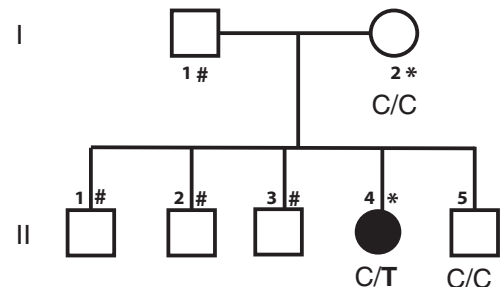



\# gDNA sample not available

Fig. 4 Pedigrees of Nigerian Yoruba families segregating likely causative variants in genes associated with syndromic forms of hearing loss. Features in addition to $\mathrm{HL}$ associated with pathogenic variants in these genes are known to have variable expressivity. Legend for pedigree drawings is similar to that of Fig. 3. The genotype for the candidate variant(s) are shown above each pedigree. + represents the reference allele; /, variants in trans.

\section{Variants identified in genes associated with nonsyndromic forms of hearing loss}

Variants predicted by bioinformatic analyses to be damaging and segregating with $\mathrm{HL}$ were identified in four genes known to be associated with recessive forms of nonsyndromic HL (Table 1, Supplementary Table 4, and Figs. 2 and 3a). A homozygous variant (confirmed by ddPCR) that is likely pathogenic was identified in CIB2 [MIM: 605564, DFNB48] in Family 2. Potential compound heterozygous variants that are pathogenic or predicted to be damaging were detected in ILDR1 [MIM: 609739, DFNB42] in Family 57 and in MYO15A [MIM: 602666, DFNB3] in Families 6, 13, and 44 (Table 1 and Fig. 3a). In addition, two damaging homozygous variants were identified in TMPRSS3 [MIM: 605511, DFNB8/B10] (NM_024022.3:c.323-6G>A, a previously reported variant, and c.1363T>C, p.(Ter455ArgextTer9)) in one subject with $\mathrm{HL}$ in Family 40. Using ddPCR, we confirmed that this subject does not carry a deletion in this region, and further showed that these variants are present within a stretch of homozygosity at the telomeric $\sim 5.3-\mathrm{Mb}$ region of chromosome $21 \mathrm{q}$. It may be a consequence of maternally inherited partial uniparental isodisomy or this individual with $\mathrm{HL}$ could have received the same region of chromosome 21 from her father, which we could not examine (gDNA not available). Analysis of the exome of this individual with $\mathrm{HL}$ did not reveal other regions of homozygosity, suggesting that she is not the result of a consanguineous mating.

Potential causative variants were identified in two genes associated with dominant forms of $\mathrm{HL}$ : in COL11A1 [MIM: 120280, DFNA37] NM_001854.4: c.1031C >T, p.(Thr344Met) in Family 23, and NM_001854.4:c.1314G>A, p.(Met438lle) in Family 32, and in WFS1 [MIM: 606201, DFNA6/A14/A38] NM_006005.3: c.2029G >A, p.(Ala677Thr) in Family 51 (Table 1, Supplementary Table 4, and Fig. $3 a$ ). These variants were not inherited maternally and paternal gDNA was not available in these three families to assess whether these variants arose de novo. Pathogenic variants in COL11A1 have been associated with nonsyndromic forms of $\mathrm{HL}$ DFNA37 [MIM: 618533] [25], and syndromic HL associated with Marshall and Stickler Syndromes [MIM: 154780 and 604841] [26]. In Family 32, a second variant predicted to be damaging,
NM_001854.4: c.4049C>G, p.(Ser1350Cys), was identified in the individual with $\mathrm{HL}$ (Table 1 and Supplementary Table 4). This variant was inherited from the mother who did not have HL. Further evaluation of this individual with $\mathrm{HL}$ for potential signs of Stickler Syndrome was not possible. Variants of WFS1 are also associated with AD Wolfram-like Syndrome and AR Wolfram Syndrome [MIM: 614296 and 222300].

In these nine families, among these rare variants predicted to be deleterious by bioinformatic approaches, only the homozygous CIB2 variant, TMPRSS3 splice variant, and potential compound heterozygous ILDR1 variants were classified as pathogenic or likely pathogenic according to ACMG/AMP guidelines for the interpretation of sequence variants in $\mathrm{HL}$ genes [22]. In the other families, one or both of the identified variants were classified as VUS (Table 1).

\section{Variants identified in genes associated with nonsyndromic forms of hearing loss or Usher syndrome}

Variants predicted to be damaging and known or suspected to be compound heterozygous were identified in $\mathrm{CDH} 23$ [MIM: 605516] in Families 8, 21, 56 (father with $\mathrm{HL}$ ), and 60 (mother with $\mathrm{HL}$ ), MYO7A [MIM: 276903] in Family 56 (mother with $\mathrm{HL}$ ), PCDH15 [MIM: 605514] in Family 5, and USH2A [MIM: 608400] in Family 29 (mother with HL) (Table 1, Supplementary Table 4, and Fig. 3b). In Family 60 (mother with $\mathrm{HL}$ ), two VUS were found in OTOF, in addition to the two VUS identified in $\mathrm{CDH} 23$ (Table 1). Overall, in these seven families with potential causal variants in genes associated with nonsyndromic HL or Usher syndrome [27, 28], the variants met both ACMG/AMP criteria for the interpretation of sequence variants in $\mathrm{HL}$ genes for pathogenic or likely pathogenic classification in three families (Families 5, 8, and 21).

\section{Variants found in genes associated with other syndromic forms of hearing loss}

We also analyzed our cohort for variants in 175 genes known to be involved in syndromic forms of HL (Supplementary Information 3), following the same strategy presented in Fig. 1. Variants predicted to be damaging were identified in CHD7 [MIM: 608892] (Families 


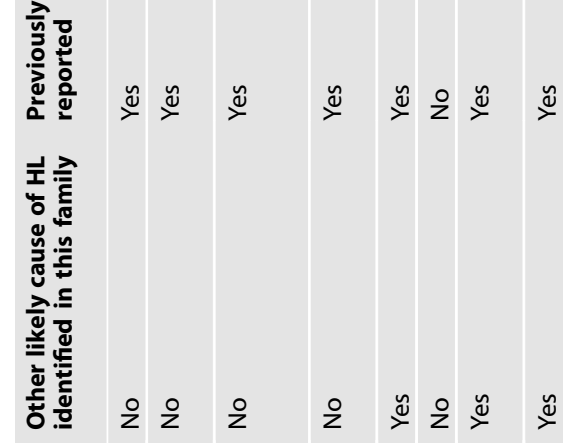
[MIM: 605712] (Family 26; Fig. 4). These variants, although classified as VUS, were all predicted to be damaging by multiple algorithms. The variants were absent in Yoruba controls and extremely rare in other populations (Table 1 and Supplementary Table 4). Pathogenic variants of $C H D 7$ are known to be associated with CHARGE syndrome [29] [MIM: 214800] and hypogonadotropic hypogonadism with or without anosmia [30] [MIM: 612370]. OPA1 pathogenic variants have been reported in patients with $A D$ optic atrophy 1 (ADOA, [MIM: 165500]), which can also be associated with delayed-onset sensorineural deafness (ADOAD) [MIM: 125250] [31, 32]. SPTLC1 pathogenic variants have been reported in patients with hereditary sensory and autonomic neuropathy type IA [MIM: 162400] [33].

\section{A few rare mitochondrial variants were identified in MT-RNR1} We used Sanger sequencing to detect pathogenic variants in three mitochondrial genes reported to be associated with HL. We identified several variants in MT-RNR1 [MIM: 561000] encoding the $12 S$ rRNA (Supplementary Table 5). Eight of those variants were absent from Yoruba control samples and rare in other populations (Table 2). Although the pathogenicity of most of these variants is unknown, the variant m.1555A>G found in Family 60 (mother with $\mathrm{HL}$ ) has previously been associated with aminoglycoside-induced $\mathrm{HL}$ as well as late-onset nonsyndromic $\mathrm{HL}$ not associated with aminoglycoside exposure [34-37]; however, other potential causes of $\mathrm{HL}$ have also been identified in the same individual (Table 1). We did not detect variants in MT-TS1 [MIM: 590080]. One variant was found in MT-TL1 [MIM: 590050]. It was predicted to be benign ( $\mathrm{m} .3277 \mathrm{G}>\mathrm{A}, \mathrm{c.48G}>\mathrm{A}$ ) and was detected in the probands and their unaffected mothers in both Families 1 and 51. In Family 1, it was also detected in one unaffected sibling.

Overall, our molecular genetic analyses indicate a remarkably high level of heterogeneity associated with childhood $\mathrm{HL}$ in Yorubas from Ibadan, Nigeria, including the presence of the mitochondrial variant associated with aminoglycoside-induced $\mathrm{HL}$ m.1555A $>$ G.

\section{DISCUSSION}

No prevalent pathogenic variants for $\mathrm{HL}$ were identified in this cohort of 56 small families of Yoruba ethno-lingual ancestry ascertained in or near Ibadan, Nigeria and segregating severe-toprofound bilateral sensorineural $\mathrm{HL}$ with congenital or early childhood onset. Only heterozygous variants of GJB2 were identified, none of which were associated in trans with any of the three previously reported deletions in the genomic region of GJB2-GJB6. These results are consistent with those reported for 44 probands from Nigeria with nonsyndromic HL [15] and 90 families from Nigeria with nonsyndromic mild-to-profound prelingual HL [14]. In addition, none of the variants we identified were previously reported in those studies, supporting our conclusion of highly diverse genetic etiology of childhood $\mathrm{HL}$ in Nigeria. The heterogeneity of variants associated with $\mathrm{HL}$ may reflect the genomic heterogeneity of Sub-Saharan Africans and the fact that the Ibadan population has lived in a large city for many centuries with a constant flux of people. For AR forms of $\mathrm{HL}$, homozygous variants were only found in a few cases, consistent with a lack of consanguineous matings in Yoruba population and the diverse array of variants identified at low frequency.

Different variants of a gene may cause either a syndromic form of HL such as Usher syndrome or nonsyndromic HL $[7,38]$. Although our recruitment focused on probands with nonsyndromic $\mathrm{HL}$, we identified several pathogenic, likely pathogenic and VUS with predicted deleterious effects in genes that can cause nonsyndromic $\mathrm{HL}$ or Usher syndrome (CDH23, MYO7A, $P C D H 15$, and USH2A), CHARGE syndrome (CHD7), syndromic optic atrophy (OPA1), and a hereditary sensory and autonomic 
neuropathy (SPTLC1). The $\mathrm{HL}$ phenotype in these individuals may indeed be nonsyndromic, but a syndromic association could have been missed due to the young age of the patients at the time of examination, before the onset of signs or symptoms such as retinitis pigmentosa as the cause of vision loss in Usher syndrome. Furthermore, some of the signs and symptoms may have been present but either subclinical or may not have been detected at the time of examination. Another hypothesis is that there are modifiers present in African populations which are not present in the populations in which these syndromes have been phenotypically characterized. Variable expressivity of clinical presentations associated with CHD7 and OPA1 variants, both within and between families, has been previously documented $[30,39]$.

In a subset of families, including Family 60 that has a mother with $\mathrm{HL}$, we identified several potential causes of HL. Lack of access to parental gDNA and limited information regarding the pathogenicity of the variants does not allow a definitive conclusion regarding the etiology of the $\mathrm{HL}$ in this family.

In $66.7 \%$ of the cases, the analyses presented here did not identify any potential causative variants in nonsyndromic and syndromic HL reported genes. This may be due to technical difficulties such as variants missed that are located in poorly sequenced GC rich coding exons, deletions of the scale of an exon and insertions that may not have been detected by our CNV analyses, failure to detect causal variants affecting splicing or in noncoding regions of the genome, unannotated exons for which there are no probes in the commercial ES reagents we used, novel $\mathrm{HL}$ genes or a nongenetic, multigenic, or multifactorial etiology of the $\mathrm{HL}$ in those families. We identified additional rare VUS with predicted deleterious effects in some of the unsolved families in this study in genes associated with syndromic forms of HL (Supplementary Table 3). Further clinical examinations of the individuals carrying such variants would be very informative, but are hampered by the difficulty of recontacting and re-phenotyping the families. In numerous families, we identified rare heterozygous pathogenic or likely pathogenic variants in genes that are associated with recessive forms of HL. The presence of these variants could be coincidental but could also indicate that we failed to identify a second pathogenic variant responsible for the phenotype.

In conclusion, our study highlights the extreme heterogeneity of variants and genes associated with $\mathrm{HL}$ in the Yoruba population of Sub-Saharan Africa. This work underscores the need for comprehensive genomic sequencing approaches for molecular genetic diagnosis of $\mathrm{HL}$ in Sub-Saharan Africans.

\section{DATA AVAILABILITY}

All variants thought to cause $\mathrm{HL}$ have been submitted to the ClinVar public database under the reference SUB10099771 (https://www.ncbi.nlm.nih.gov/clinvar/).

\section{REFERENCES}

1. Morton CC, Nance WE. Newborn hearing screening-a silent revolution. N Engl J Med. 2006;354:2151-64.

2. Watkin $\mathrm{P}$, Baldwin M. The longitudinal follow up of a universal neonatal hearing screen: the implications for confirming deafness in childhood. Int J Audio. 2012;51:519-28.

3. Mulwafu W, Kuper $H$, Ensink RJ. Prevalence and causes of hearing impairment in Africa. Trop Med Int Health. 2016;21:158-65.

4. Olusanya BO, Wirz SL, Luxon LM. Community-based infant hearing screening for early detection of permanent hearing loss in Lagos, Nigeria: a cross-sectional study. Bull World Health Organ. 2008;86:956-63.

5. Olusanya BO. Newborns at risk of sensorineural hearing loss in low-income countries. Arch Dis Child. 2009;94:227-30.

6. Olusanya BO, Emokpae A, Renner JK, Wirz SL. Costs and performance of early hearing detection programmes in Lagos, Nigeria. Trans R Soc Trop Med Hyg. 2009;103:179-86.
7. Griffith AJ, Friedman TB. Hereditary hearing loss. In: Wackym PA, Snow JB Jr, editors. Ballenger's otorhinolaryngology head and neck surgery. 18th ed. Shelton, CT: People's Medical Publishing House-USA, Ltd; 2016. p. 329-45.

8. Dror AA, Avraham KB. Hearing loss: mechanisms revealed by genetics and cell biology. Annu Rev Genet. 2009;43:411-37.

9. Chukuezi AB. Profound and total deafness in Owerri, Nigeria. East Afr Med J. 1991;68:905-12.

10. Obiako MN. Profound childhood deafness in Nigeria: a three year survey. Ear Hear. 1987;8:74-7.

11. Labaeka AA, Tongo OO, Ogunbosi BO, Fasunla JA. Prevalence of hearing impairment among high-risk newborns in Ibadan, Nigeria. Front Pediatr. 2018; 6:194.

12. Lasisi AO, Onakoya PA, Lasisi TJ, Akinola MD, Tongo O. Neonatal hearing screening in a rural/sub-urban community in Nigeria, sub-Saharan Africa-a preliminary report. Int J Pediatr Otorhinolaryngol. 2014;78:1452-5.

13. Rudman JR, Kabahuma Rl, Bressler SE, Feng Y, Blanton SH, Yan D, et al. The genetic basis of deafness in populations of African descent. J Genet Genomics. 2017;44:285-94.

14. Yan D, Tekin D, Bademci G, Foster J 2nd, Cengiz FB, Kannan-Sundhari A, et al. Spectrum of DNA variants for non-syndromic deafness in a large cohort from multiple continents. Hum Genet. 2016;135:953-61.

15. Lasisi AO, Bademci G, Foster J 2nd, Blanton S, Tekin M. Common genes for nonsyndromic deafness are uncommon in sub-Saharan Africa: a report from Nigeria. Int J Pediatr Otorhinolaryngol. 2014;78:1870-3.

16. Del Castillo I, Moreno-Pelayo MA, Del Castillo FJ, Brownstein Z, Marlin S, Adina Q, et al. Prevalence and evolutionary origins of the del(GJB6-D13S1830) mutation in the DFNB1 locus in hearing-impaired subjects: a multicenter study. Am J Hum Genet. 2003;73:1452-8.

17. Wilch E, Azaiez H, Fisher RA, Elfenbein J, Murgia A, Birkenhager R, et al. A novel DFNB1 deletion allele supports the existence of a distant cis-regulatory region that controls GJB2 and GJB6 expression. Clin Genet. 2010;78:267-74.

18. Schultz JM, Khan SN, Ahmed ZM, Riazuddin S, Waryah AM, Chhatre D, et al. Noncoding mutations of HGF are associated with nonsyndromic hearing loss, DFNB39. Am J Hum Genet. 2009;85:25-39.

19. Mencia A, Modamio-Hoybjor S, Redshaw N, Morin M, Mayo-Merino F, Olavarrieta $\mathrm{L}$, et al. Mutations in the seed region of human miR-96 are responsible for nonsyndromic progressive hearing loss. Nat Genet. 2009;41:609-13.

20. Shearer AE, Eppsteiner RW, Booth KT, Ephraim SS, Gurrola J 2nd, Simpson A, et al. Utilizing ethnic-specific differences in minor allele frequency to recategorize reported pathogenic deafness variants. Am J Hum Genet. 2014;95:445-53.

21. Schrauwen I, Liaqat K, Schatteman I, Bharadwaj T, Nasir A, Acharya A, et al. Autosomal dominantly inherited GREB1L variants in individuals with profound sensorineural hearing impairment. Genes (Basel). 2020;11:687.

22. Oza AM, DiStefano MT, Hemphill SE, Cushman BJ, Grant AR, Siegert RK, et al. Expert specification of the ACMG/AMP variant interpretation guidelines for genetic hearing loss. Hum Mutat. 2018;39:1593-613.

23. DiStefano MT, Hemphill SE, Oza AM, Siegert RK, Grant AR, Hughes MY, et al. ClinGen expert clinical validity curation of 164 hearing loss gene-disease pairs. Genet Med. 2019;21:2239-47.

24. Hindson BJ, Ness KD, Masquelier DA, Belgrader P, Heredia NJ, Makarewicz AJ, et al. High-throughput droplet digital PCR system for absolute quantitation of DNA copy number. Anal Chem. 2011;83:8604-10.

25. Booth KT, Askew JW, Talebizadeh Z, Huygen PLM, Eudy J, Kenyon J, et al. Splicealtering variant in COL11A1 as a cause of nonsyndromic hearing loss DFNA37. Genet Med. 2019;21:948-54.

26. Khalifa O, Imtiaz F, Ramzan K, Allam R, Hemidan AA, Faqeih E, et al. Marshall syndrome: further evidence of a distinct phenotypic entity and report of new findings. Am J Med Genet A. 2014;164A:2601-6.

27. Friedman TB, Griffith AJ. Human nonsyndromic sensorineural deafness. Annu Rev Genomics Hum Genet. 2003;4:341-402.

28. Geleoc GGS, El-Amraoui A. Disease mechanisms and gene therapy for Usher syndrome. Hear Res. 2020;394:107932.

29. Janssen N, Bergman JE, Swertz MA, Tranebjaerg L, Lodahl M, Schoots J, et al. Mutation update on the CHD7 gene involved in CHARGE syndrome. Hum Mutat. 2012;33:1149-60.

30. Kim HG, Kurth I, Lan F, Meliciani I, Wenzel W, Eom SH, et al. Mutations in CHD7, encoding a chromatin-remodeling protein, cause idiopathic hypogonadotropic hypogonadism and Kallmann syndrome. Am J Hum Genet. 2008;83:511-9.

31. Yu-Wai-Man P, Griffiths PG, Gorman GS, Lourenco CM, Wright AF, Auer-Grumbach $M$, et al. Multi-system neurological disease is common in patients with OPA1 mutations. Brain. 2010;133:771-86.

32. Amati-Bonneau P, Guichet A, Olichon A, Chevrollier A, Viala F, Miot S, et al. OPA1 $\mathrm{R} 445 \mathrm{H}$ mutation in optic atrophy associated with sensorineural deafness. Ann Neurol. 2005;58:958-63. 
33. Nicholson GA. SPTLC1-related hereditary sensory neuropathy. In: Adam MP, Ardinger $\mathrm{HH}$, Pagon RA, Wallace SE, Bean LH, Mirzaa G, et al., editors. GeneReviews((R)). Seattle, WA: University of Washington; 1993.

34. Prezant TR, Agapian JV, Bohlman MC, Bu X, Oztas S, Qiu WQ, et al. Mitochondrial ribosomal RNA mutation associated with both antibiotic-induced and non-syndromic deafness. Nat Genet. 1993;4:289-94.

35. Fischel-Ghodsian N, Prezant TR, Bu X, Oztas S. Mitochondrial ribosomal RNA gene mutation in a patient with sporadic aminoglycoside ototoxicity. Am J Otolaryngol. 1993;14:399-403.

36. Hutchin T, Haworth I, Higashi K, Fischel-Ghodsian N, Stoneking M, Saha N, et al. A molecular basis for human hypersensitivity to aminoglycoside antibiotics. Nucleic Acids Res. 1993;21:4174-9.

37. Bacino C, Prezant TR, Bu X, Fournier P, Fischel-Ghodsian N. Susceptibility mutations in the mitochondrial small ribosomal RNA gene in aminoglycoside induced deafness. Pharmacogenetics. 1995;5:165-72.

38. Bademci G, Cengiz FB, Foster li J, Duman D, Sennaroglu L, Diaz-Horta O, et al. Variations in multiple syndromic deafness genes mimic non-syndromic hearing loss. Sci Rep. 2016;6:31622.

39. Jongmans MC, Hoefsloot LH, van der Donk KP, Admiraal RJ, Magee A, van de Laar I, et al. Familial CHARGE syndrome and the CHD7 gene: a recurrent missense mutation, intrafamilial recurrence and variability. Am J Med Genet A. 2008;146A:43-50.

\section{ACKNOWLEDGEMENTS}

We thank the participants in this study and Drs D. Drayna and S. Naz for their comments on the manuscript. The following cell lines/DNA samples were obtained from the NHGRI Sample Repository for Human Genetic Research at the Coriell Institute for Medical Research: [MGP00013]. This study utilized the computational resources of the NIH HPC Biowulf cluster (http://hpc.nih.gov) at the National Institutes of Health, Bethesda, MD.

\section{AUTHOR CONTRIBUTIONS}

$A A, T B F$, and AJG conceived the study and its initial design. AA and SO recruited the patients, performed their clinical assessment, and obtained their gDNA. RF and IR performed exome library preparation. RJM provided exome sequencing results. RF, PC, RY, RT, TB, LMNS, AnA, IS, RJM, SML, TBF, AJG, and IR analyzed exome sequencing data. RF, PC, RY, and RT Sanger confirmed the variants identified and studied their family segregation. PC Sanger sequenced the mitochondria genes analyzed and checked variant MAF in Yoruba samples from the Coriell. RY performed ddPCR analysis. IR, RF, AA, SL, IS, TBF, and AJG wrote the first draft of the manuscript. All authors reviewed, commented, and agreed on the final draft of the manuscript.

\section{FUNDING}

This work was funded (in part) by the National Institute on Deafness and Other Communication Disorders (NIDCD) (NIH Intramural Research Funds ZIC DC000086 to RJM, DC000039 to TBF, and DC000060 to AJG and IR) as well as extramural NIDCD funding DC011651, DC016593, and DC003594 to SML.

\section{COMPETING INTERESTS}

The authors declare no competing interests.

\section{ADDITIONAL INFORMATION}

Supplementary information The online version contains supplementary material available at https://doi.org/10.1038/s41431-021-00984-w.

Correspondence and requests for materials should be addressed to Adebolajo Adeyemo or Isabelle Roux.

Reprints and permission information is available at http://www.nature.com/ reprints

Publisher's note Springer Nature remains neutral with regard to jurisdictional claims in published maps and institutional affiliations.

Open Access This article is licensed under a Creative Commons c. Attribution 4.0 International License, which permits use, sharing, adaptation, distribution and reproduction in any medium or format, as long as you give appropriate credit to the original author(s) and the source, provide a link to the Creative Commons license, and indicate if changes were made. The images or other third party material in this article are included in the article's Creative Commons license, unless indicated otherwise in a credit line to the material. If material is not included in the article's Creative Commons license and your intended use is not permitted by statutory regulation or exceeds the permitted use, you will need to obtain permission directly from the copyright holder. To view a copy of this license, visit http://creativecommons. org/licenses/by/4.0/.

This is a U.S. government work and not under copyright protection in the U.S.; foreign copyright protection may apply 2021 